\title{
Research Paper: The Effects of 10-Week Aerobic-cognitive Combined Training on Serum Levels of High Sensitivity C-reactive Protein, Anti-cardiolipin antibodies, and Mental Status of Female Patients With Lupus Erythematosus Systemic: A Preliminary Study
}

Zahra Azarpira $^{1^{*}}$ (D), Hamid Rajabi ${ }^{1}$ (D), Afkham Daneshfar ${ }^{2}$ (iD, Seyedeh Tahereh Faezi ${ }^{3}$

1. Department of Exercise Physiology, Faculty of Physical Education and Sport Sciences, Kharazmi University, Tehran, Iran.

2. Department of Motor Behavior, Faculty of Sport Sciences, Alzahra University, Tehran, Iran.

3. Rheumatology Research Center, Shariati Hospital, Tehran University for Medical Sciences, Tehran, Iran.

\begin{tabular}{l|l}
\hline $\begin{array}{l}\text { use your device toscan } \\
\text { and read the article online }\end{array}$ & $\begin{array}{l}\text { High Sensitivity C-reactive Protein, Anti-cardiolipin antibodies, and Mental Status of Female Patients With Lupus Erythematosus Systemic: } \\
\text { APreliminary Study. Journal of Modern Rehabilitation. 2018; 12(4):225-232 http://dx.doi.org/10.32598/JMR.V12.N4.225 }\end{array}$ \\
dolo http://dx.doi.org/10.32598/JMR.V12.N4.225
\end{tabular}

\section{(i) (3)}

Article info:

Received: 25 Jan 2018

Accepted:13 Aug 2018

Available Online: 01 Oct 2018

Keywords:

Aerobic exercise, Cognitive functions, Mini-mental state examination, Inflammatory factors

\section{A B S T RACT}

Introduction: Physical training, especially aerobic exercise combined with mental training by patients with Systemic Lupus Erythematosus (SLE), has numerous benefits, including tissue generation and enhanced anti-inflammatory activity. This study aimed at investigating the effect of ten-week aerobic-cognitive combined training on the serum levels of High Sensitivity C-Reactive Protein (HS-CRP), Anti-cardiolipin antibodies, Immunoglobulin G (IgG), Immunoglobulin M (IgM), and mental status of female SLE patients.

Materials and Methods: Eight female patients with SLE (with the Mean \pm SD age of $50 \pm 47.04$ year and the Mean \pm SD BMI of $29.57 \pm 5.83 \mathrm{~kg} / \mathrm{m}^{2}$ ) were randomly assigned to the experimental $(\mathrm{n}=5)$ and control $(\mathrm{n}=3)$ groups. The experimental group performed Brailletonik exercise, including aerobic (60-80\% of Max HR) and cognitive training for ten weeks. The serum levels of HS-CRP, IgG, IgM, and mental status of the patients were evaluated in both groups before and after the training.

Results: According to the results of $2 \times 2$ mixed Analysis of Covariance (ANCOVA), there was no significant effect of training on the serum levels of HS-CRP, IgG, and IgM $(\mathrm{P}>0.05)$, but HSCRP and IgM level decreased in the experimental group by (42\%) and (23.5\%), respectively. The result of ANCOVA indicated the significant effect of training on mental status $(\mathrm{P}=0.005)$.

Conclusion: Physical combined cognitive training could be more effective in improving the mental status compared to a decrease in serum inflammatory factors of SLE patients. 


\section{Introduction}

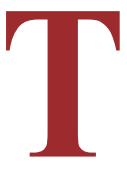

he Systemic Lupus Erythematosus (SLE) is a chronic, systemic, relapsing-remitting autoimmune inflammatory disease, in which the immune system is activated against the body cells leading to tissue damage. The prevalence of this disease is nine times higher in women. Usually, people in the age range of 1545 years are diagnosed with this disease. The involvement of the Central Nervous System (CNS) is one of the first known aspects of this disease [1].

Based on a study by Rheumatologic Research Center of Tehran University of Medical Sciences, the prevalence of lupus is 40 per 100000; this means that out of every 2500 Iranians, one has lupus. The symptoms of this disease could be mild or severe, depending on the patient [2]. One of the major characteristics of lupus is its periodic occurrence, including recurrence (intensified symptoms) and quenched (reduced symptoms) phases [3]. Two types of lupus have been diagnosed so far; dermatologic erythematosus lupus, which only affects the patient skin, and Systematic Lupus Erythematosus (SLE), which is an autoimmune disease accompanied by the inflammation of epithelial tissue and vessels with the ability to involve almost all of the body organs, including joints, skin, kidneys, and heart [4].

Various factors are effective in SLE, among which environmental, immunologic, and genetic factors are more important. These factors stimulate the immune response, which results in the production of antibodies against the cell antigens and, hence, reduces the tolerance of the immune system [5]. They can also cause the formation of the immune complexes, which can remain in the body and decrease the immune system function leading to systematic inflammatory progress. Although the exact etiology of SLE is still unclear, a wide range of studies and development have been conducted and made in this field. The two major disorders of this disease are immunologic disorders and self-reactive antibodies [6].

Studies have shown that systematic inflammation level is high in lupus patients as it is an autoimmune disease, one of whose features is the chronic inflammation. High levels of High Sensitivity C-Reactive Protein (HS-CRP), Interleukin (IL-10), (IL-6), (IL-4), Tumor necrosis factor (TNF)- $\alpha$, (IL-12), and Interferon (IFN)- $\gamma$ have been observed in these patients, which are regarded as the main inflammatory factors of SLE pathogenesis [7-10].
Neuropsychiatric disorders are detected in more than half of lupus patients; they could be manifested in various forms, including motor disorders, nerve demyelinating, cognitive disorders, dizziness, mood disorders, depression, anxiety, epilepsy, and psychosis [11, 12]. Mild levels of cognitive disorders are more common in lupus patients, whose attention, visual and speech memory, motor function, reaction speed, and motor perception could be damaged; Though clinical symptoms are extensive and independent of disease, and no standard test has yet been established to diagnose Neuropsychiatric Systemic Lupus Erythematosus (NPSLE) [13]. Several pathologic pathways are, however, determined, which correspond with clinical symptoms of lupus; for example, neurotoxicity mediator antibody, anti-phospholipid antibodies and anti-cardiolipin antibodies (Immunoglobulin $\mathrm{G}$ [IgG], Immunoglobulin M [IgM], and Immunoglobulin [IgA]) [14]. The latter is a group of immunoglobulins created against membrane phospholipids giving rise to blood coagulation. Some researchers have tried to associate these antibodies (which only exist in 30-40\% of SLE patients) with cognitive impairments and a wide range of psychologicalneural disorders in NPSLE $[15,16]$.

In a study by Sato et al. on NPSLE patients, the increase in the level of pro-inflammatory cytokines and antibodies and albumins were detected in their cerebrospinal fluid [17]. Planting et al. also investigated the effect of aging on physical and cognitive performance of SLE patients. They selected 60 adult SLE patients ( $90 \%$ female) in various age ranges $20-39(26.7 \%), 40-$ $59(50 \%)$, and over $60(23.3 \%$ ) years old; $80 \%$ of them were black. They evaluated their cognitive functions and motor perception. Their results showed lupus inflammation-induced damages in all age ranges, especially at older ages [18].

Among the interventions for SLE, physical training can play an active role in enhancing the cognitive functions of lupus patients and Protocols conducted on mice showed that a combination of physical exercise and environmental enrichment could result in sensorymotor stimulation, reduced brain cortex inhibition, and the better perceptual and cognitive abilities mediated through the increase of cell proliferation, neurogenesis, and gliogenesis in the hippocampus [19]. This could be owing to an increase in local blood circulation and generation of new nervous cells [20].

Human studies also confirmed the relationship between regular physical activities and brain development, especially in the premotor brain cortex. Regular 
physical activities control the growth and differentiation of nervous neurons, synaptogenesis, and arteriogenesis, which will improve neurotrophy and, hence, enhance the cognitive function such as processing speed, motor perception, control, and working memory [21, 22].

The anti-inflammatory role of physical activities in SLE disease has also been studied by some researchers [23]. Rheumatologic studies have shown that exercise can have a positive effect on the quality of life of patients with lupus by reducing pain and stiffness of the muscles, as well as reducing the frequency of the recurrence of chronic pain and improving the body performance and cognitive function of the patients. Muller and Perado et al. confirmed that increased physical activities were a helpful method in the treatment of SLE, It was also determined that increasing physical training could rise maximum oxygen consumption, aerobic capacity, autonomic cardiac control, and quality of life of SLE patients in all age ranges and It has been hypothesized that physical activities can enhance the severity of this disease by releasing pro-inflammatory response. This hypothesis is, however, in contrast with the anti-inflammatory effect of physical training in chronic diseases with partial systematic inflammation (such as type II diabetes) [24].

According to the studies, physical activity is one of the active factors in changing the immune system function, which is highly dependent on the intensity, duration, and type of the training, as well as physical fitness, nutrition, mental state, and hormonal factors and thus, guiding the patients to perform sports programs along with pharmacotherapy and conducting applicable exercises to enhance their functional and mental abilities can control or prevent NPSLE-induced inflammatory and cognitive impairments. Leonorth et al. conducted a systematic review of the effect of combinational training (physical and cognitive) on cognitive function and They reported that cognitive and physical training should be combined to improve performance. In most of the studies in their review, combinational training affected the cognitive function [25].

No study has yet addressed the effect of aerobic-cognitive combined training on the inflammatory indicators and mental status of SLE patients. Moreover, given the impact of hormones on the emergence and progress of lupus and its higher prevalence among women (more than twice), further studies in female patients with SLE are necessary [26]. Also, in most of the previous studies, aerobic and cognitive training have been used, but since brailletonik is an Iranian cognitive-motor training, in which movements are based on international alphabetical codes, in this study, we used Brailletonik for the aerobic-cognitive combined training. In this regard, the present study investigated the effect of ten-week aerobic-cognitive training on the serum levels of HS-CRP, $\mathrm{IgG}$, and $\mathrm{IgM}$, as well as the mental status of female patients with SLE.

\section{Materials and Methods}

This study was conducted on eight female patients with SLE with the mean age of 50 years and the mean BMI of $29.57 \mathrm{~kg} / \mathrm{m}^{2}$. They were selected based on convenience sampling method from the members of the Iran Lupus Association and were randomly assigned to the experimental $(n=5)$ and control $(n=3)$ groups. The participants were, then, evaluated in terms of disease severity using Systemic Lupus Erythematosus Disease Activity Index (SLEDAI) questionnaire, disease duration, and psychological diseases, such as epilepsy, psychosis, and depression (using Beck depression inventory). Their motor balance was also assessed by a rheumatologist.

The participants were homogenized in terms of factors effective in inflammation and cognitive aspects. We used the pre-test, post-test randomized-group design for this study, in which the motor-cognitive brailtonic training was performed for ten weeks (three sessions per week) under the supervision of the researcher. The serum levels of HS-CRP, IgM, and IgG, as well as the mental status of the patients by the Mini-mental State Examination (MMSE) questionnaire, were assessed in pretest and posttest. It is worth noting that all stages of the research, from participants' selection and training steps to sampling, were conducted under the supervision of Iran Lupus Association.

First, the maximum heart rate of each participant was calculated (220 minus age of the participant). Next, they were trained to measure their heart rate by palpating their neck carotid artery. They measured their resting and activity heartbeat number per minute before and after each session under the supervision of the researcher, which were recorded in the training session checklist. According to the type of protocol that is continuously increasing and based on the performance of the subjects in the first session, the initial training intensity $(60 \%$ of maximum heart rate) was determined. Then, the principles of overload were added weekly to the intensity and duration of the training.

Training protocol included ten successive weeks (30 sessions) of exercise (three 60-minute sessions per 
week). The training was a combination of cognitive and aerobic exercises with an intensity of (60-80\%) of Max HR of the participants. In the first week and then in the second, third, and fourth weeks, the participants worked out (60\%) and (70\%) of their Max HR, respectively. They exercised with $(80 \%)$ of their Max HR in the remaining 4 weeks.

Each training session included 10 to 15 minutes of dynamic and aerobic warm-up, stretching movements, and 40 minutes of brailtonic training, which included responding to auditory and visual stimuli, fast processing (the speed, at which the brain receives new information), comprehension, attention, memory (including both chained skills and long-term memory) and logic and reasoning (the ability to deduce and think in a sequence and rational order). The motor-cognitive brailtonic training was performed by a 6-cell table and braille signs for alphabetical coding limbs movements based on the codes and recalling the moves from memory.

The six-cell table can be placed on the floor, wall, or even can be imagined in the space. Brailtonic training starts with the movements on table numbers. Then, the braille sign of each letter of the alphabet was taught, and the movements according to each code were displayed. The chosen motions for brailtonic training are methods of enhancing mental-cognitive skills. The designed training include hand stretching, balance movements, contentious movements for hands and legs, gait movements, rotation, mimic movements from the leader, and mild jumps.

At the end of each training session, cool-down training was done for ten minutes (slow walking and stretching) with intensity less than the initial intensity of each session to recover the initial condition. The control group had no physical activity and continued an inactive lifestyle.
To analyze the research variables, before the test and then after the test (48 hours after the last training session), ten $\mathrm{mL}$ sample venous blood was taken from the left hand of the participants after 12 hours of fasting and five minutes of rest, using sterile Venoject syringes containing ethylenediaminetetraacetic acid anti-coagulation agents. Then, the samples were put in an ice bath. The serum was separated, by centrifuging and kept at (-20) for further analysis. To determine the HS-CRP level, the specific kit of HS-CRP assessment (MININEPH, Binding Site Group, UK) was used. In a typical procedure, the serum samples were placed in a nephelometer instrument (which uses light scattering to measure the protein concentration), a specific amount of anti-serum was, then, added, resulting in antibody-antigen interactions.

The device measured the reference light, which was proportional to the antigen-antibody complex level at the wavelength of $670 \mathrm{~nm}$; hence, the concentration of the $\mathrm{C}$-protein could be assessed. To measure serum immunoglobulins (IgG and $\operatorname{IgM}$ ), nephelometer instrument, and specific kits (Binding Site Group, UK) were applied.

According to the results of the Shapiro-Wilk test, the distribution of the data was normal $(\mathrm{P}>0.05)$. Also, there was a significant difference between the weight of the two groups based on t-test ( $\mathrm{t} 6=-2.635, \mathrm{P}=0.039$ ). Thus, to analyze the data of HS-CRP, IgG, and IgM, two (group) $\times$ two (time) analysis of covariance $(\mathrm{AN}$ COVA) with the repeated measures of the last factor and weight as a covariate was used. The data of MMSE were analyzed by ANCOVA with weight and pre-test as covariates. The significance level in all analyses was considered less than 0.05 .

Table 1. Mean $\pm S D$ of study variables in the experimental and control groups

\begin{tabular}{ccccc}
\hline & \multicolumn{3}{c}{ Mean \pm SD } \\
\cline { 2 - 5 } Variable & \multicolumn{2}{c}{ Experimental } & \multicolumn{2}{c}{ Control } \\
\cline { 2 - 5 } & Pre-test & Post-test & Pre-test & Post-test \\
\cline { 2 - 5 } HS-CRP & $1.82 \pm 1.39$ & $1.06 \pm 0.94$ & $1.30 \pm 1.47$ & $2.63 \pm 1.71$ \\
\hline IgG & $5.85 \pm 4.18$ & $6.08 \pm 3.35$ & $8.40 \pm 7.53$ & $10.80 \pm 3.12$ \\
\hline IgM & $6.77 \pm 5.06$ & $5.18 \pm 1.45$ & $5.77 \pm 2.64$ & $6.87 \pm 1.79$ \\
\hline
\end{tabular}


Table 2. The results of $2 \times 2$ mixed ANCOVA for HS-CRP, IgG, and IgM

\begin{tabular}{ccccccc}
\hline Variable & Effect & $\mathbf{F}$ & Hypothesis df & Error df & $\mathbf{P}$ & Partial Eta Squared \\
\hline \multirow{3}{*}{ HS-CRP } & Group & 0.047 & 1 & 5 & 0.837 & 0.009 \\
& Time & 0.311 & 1 & 5 & 0.601 & 0.058 \\
& Group $\times$ time & 0.622 & 1 & 5 & 0.466 & 0.111 \\
IgG & Group & 0.581 & 1 & 5 & 0.480 & 0.104 \\
& Time & 0.057 & 1 & 5 & 0.821 & 0.011 \\
Group & Group & 0.975 & 1 & 5 & 0.959 & 0.001 \\
& Time & 0.02 & 1 & 5 & 0.303 & 0.209 \\
& Group $\times$ time & 0.347 & 1 & 5 & 0.892 & 0.004 \\
\hline
\end{tabular}

Table 3. Percentage changes of variables in the experimental and control groups

\begin{tabular}{ccccc}
\hline Variable & Experimental & \multicolumn{2}{c}{ Control } \\
\hline HS-CRP & Decrease & 42 & Increase & 101.5 \\
IgG & Increase & 4 & Increase & 28.6 \\
IgM & Decrease & 23.5 & Increase & 19.1 \\
MMSE & Increase & 15.7 & Decrease & 1.2 \\
\hline
\end{tabular}

\section{Results}

Table 1 presents the Mean \pm SD of the investigated variables in the experimental and control groups. The results of two (group) $\times$ two (time) ANCOVA with the repeated measures of the last factor and weight as covariate indicated the main effects, and the interaction of group and time was not significant for HS-CRP, IgG, and IgM (Table 2). CRP and IgM decreased (42\%) and (23.5\%), respectively, in the experimental group, while increased in the control group and the percentage increase of IgG in the experimental group (4\%) was less than that in the control group (Table 3). The result of ANCOVA with weight and pre-test as covariates for mental status indicated the significant effect of group, and the post-test score of MMSE in the experimental group was significantly more than that of the control group $\left(\mathrm{F}_{1.4}=30.896\right.$, $\mathrm{P}=0.005$, partial $\mathrm{h}_{2}=0.885$ ).

\section{Discussion}

The present study aimed to investigate the effect of 10week combined aerobic-cognitive training on HS-CRP, $\mathrm{IgG}$, IgM serum levels, as well as the mental state of SLE patients. The results indicated that the training did not significantly affect the inflammatory factor of HSCRP in the experimental group $(\mathrm{P}>0.05)$. But, the percentage change revealed that the HS-CRP level of the experimental group reduced to $(42 \%)$, which could be as a result of regular physical training and its substantial anti-inflammatory effects.

Recent reviews on the anti-inflammatory effects of regular training have focused on three possible mechanisms; the reduction in visceral fat mass, the increased production and release of anti-inflammatory cytokines (myokine) from contracting skeletal muscle, and the reduced expression of Toll-like receptors on monocytes and macrophages (with the subsequent inhibition of downstream responses such as the production of proinflammatory cytokines) [27]. 
Small sample size can influence the results of the research, and an increase in sample size could enhance the accuracy and validity of the results. In this study, the duration and intensity of training and the initial level of the participants could also affect the results. Probably, the duration and intensity were not enough to develop proper adaption. Hence, more studies are required in these training factors.

IgG analysis also indicated that training was not significantly effective in this inflammatory factor among the members of the experimental group. But, according to Table 3, the post-test values of IgG showed an increase in both groups, which may be attributable to disease activities. But, the lower percentage increase in the experimental group (4 vs. 28.6) could be attributed to the positive and anti-inflammatory impacts of training on the patients. Furthermore, the low number of participants could affect the results in a way that the between-group difference was not significant. Regarding the cognitive and physical disabilities of the patients with SLE, disease severity, pain, and movement limitations, it is not surprising that a few patients participated in the research.

Moreover, the duration of the training could influence the results and investigation of the effect of physical training on IgG inflammation factor in the more extended period will result in more accurate findings. Chronic inflammation as a result of several years of the disease could also nullify the effects of physical training. Overall, it can be concluded that although physical training has a positive impact on the disease path, its effect on IgG inflammation factor was not significant in this study.

In case of IgM variable, the training was not significantly effective in this inflammatory factor of the experimental group, but the investigation of the percentage change revealed that in the control group, IgM increased $(19.1 \%)$, while it declined $(23.5 \%)$ in the experimental group. The reason could be the anti-inflammatory effect of physical exercise on anti-cardiolipin levels in SLE patients. Furthermore, the result of IgM and the insignificant between-group difference could be attributed to the low number of participants. However, given the physical capacity and various disease severities and, hence, declined range of motion in lupus patients, Therefore, this duration (ten weeks) was considered. Persistent inflammation as a result of long-term disease and associated disability could also diminish the effects of a physical training program.

The results of the MMSE score in the experimental and control groups showed the significant effect of the train- ing on the mental status of the lupus patients $(\mathrm{P}=0.005)$. It seems that owing to neural overlapping, the physical and mental training could influence the cognitive results.

Different studies have shown that the combination of physical and cognitive training increases the sensorymotor stimulation and, thus, decreases cerebral cortex inhibition. It also improves memory abilities by enhancing neurogenesis and synaptogenesis. In this study, we used aerobic-cognitive combined training. Aerobic training improves the cardiovascular system, aerobic capacity, and subsequently, increases the local blood flow and produces new nervous cell (neurogenesis); moreover, it increases synaptogenesis especially in the hippocampus, which prevents hippocampus atrophy and memory damage; as a result, the cognitive ability of lupus patients is developed and because of the development of the capillary network, aerobic capacity, maximum oxygen consumption, the general fatigue and pain of lupus patients would reduce, which improves their cognitive ability by recovering their quality of life. In addition, active muscles secrete myokine, which reduces the activity of $\mathrm{B}$ and $\mathrm{C}$ cells, inflammation and immune responses, and, consequently, increases cognitive function of the patients [28].

In the present study, cognitive training was implemented in the form of targeted and planned aerobic training called brailtonic exercises, because in these exercises, attention, work memory, spatial visualization, reaction time, and even processing speed are developed. In addition, it seems that the combination of aerobic and cognitive training in the form of brailtonic exercises improves the function of brain neurons and the cognitive ability by creating specials conditions. These conditions include physiological changes affecting the brain, such as angiogenesis and synaptogenesis, as well as creating an enriched environment for the brain to be used repeatedly and continuously [29, 30]. Also, the results of the research indicate that the cognitive abilities of the subjects increased, so it seems that the combined training increases the involvement of different parts of the brain compared with doing aerobic or cognitive training separately [31].

The results of this study emphasize the consideration of individual differences in cognitive studies. In a study by Laura et al. the effect of simultaneous mental and aerobic training was investigated in 126 elderlies with cognitive disorders for 12 weeks. The results indicated improvements in physical and cognitive function of the participants [32]. Concerning the efficacy of cognitive training on the brain function of lupus patients, Laurance et al 
investigated the effect of aerobic and cognitive training (simultaneous or following each other) on elderlies. They studied 42 adults, who participated in 12 sessions of aerobic-cognitive training and randomly divided them into two groups of simultaneous (aerobic and cognitive training) and successive (cognitive training after aerobic exercises) training.

The results indicated an increase in spatial and verbal memory of both groups. In the successive group, however, the working memory showed significant improvement [33]. The percentage change, which was mentioned in the previous sections, indicated that the effect of training on the mental status of the patients was significant; but, the inflammatory factors did not show a considerable change with improvement. In conclusion, 10 weeks of aerobic-cognitive combined training in the present study had a significant positive effect on mental status and but not a significant impact on HS-CRP, IgM, and IgG of the participants. Thus, it seems that the combined physical-cognitive training could be more effective in improving mental state than decreasing serum inflammatory factors of patients with SLE.

\section{Ethical Considerations}

\section{Compliance with ethical guidelines}

This study approved in the research committee with the ethical code of IR.MODARES.REC.1397.239 from the Ethics Committee of Faculty of Medical Sciences, Tarbiat Modares University, the written consent of the patients to participate in the research was attained.

Funding

This paper was extracted from Phd thesis of the first author Department of Exercise Physiology, Faculty of Physical Education and Sport Sciences, Kharazmei University

\section{Authors contributions}

Conceptualization, methodology, writing-review \& editing, supervision: Zahra Azarpira, Hamid Rajabi, Afkham Daneshfar; Investigation, writing-original draft, funding acquisition, resources: Zahra Azarpira.

\section{Conflict of interest}

The authors declared no conflict of interests.

\section{Acknowledgements}

The authors of the manuscript appreciate the Iran Lupus Association for their cooperation.

\section{References}

[1] Lahita L. Systemic Lupus Erythematosus. Amsterdam: Elsevier; 2011. [DOI:10.1016/B978-0-12-374994-9.10036-1]

[2] Jeltsch-David H, Muller S. Neuropsychiatric systemic lupus erythematosus: Pathogenesis and biomarkers. Nature Reviews Neurology. 2014; 10(10):579-96. [DOI:10.1038/nrneurol.2014.148] [PMID]

[3] Akbarian M, Faezi ST, Hakemi MS, Ghiyasi M, MohammadZadeh Honarvar N, Poorgharib Shahi MH. [Systemic lupus erythematosus (Persian)]. Tehran: Andishe Arian., 2013.

[4] Yu SL, Kuan WP, Wong CK, Li EK, Tam LS. Immunopathological roles of cytokines, chemokines, signaling molecules, and pattern-recognition receptors in systemic lupus erythematosus. Clinical and Developmental Immunology. 2012 2012(715190):1-14. [DOI:10.1155/2012/715190] [PMID] [PMCID]

[5] Fu SM, Deshmukh US, Gaskin F. Pathogenesis of systemic lupus erythematosus revisited 2011: End organ resistance to damage, autoantibody initiation and diversification, and HLA-DR. Journal of Autoimmunity. 2011; 37(2):104-12. [DOI:10.1016/j.jaut.2011.05.004] [PMID] [PMCID]

[6] Ginzler EM, Dvorkina O. Newer therapeutic approaches for systemic lupus erythematosus. Rheumatic Diseases Clinics of North America. 2005; 31(1):315-28. [DOI:10.1016/j. rdc.2005.01.003] [PMID]

[7] Muñoz LE, Janko C, Schulze C, Schorn C, Sarter K, Schett $\mathrm{G}$, et al. Autoimmunity and chronic inflammation-two clearance-related steps in the etiopathogenesis of SLE. Autoimmunity Reviews. 2010; 10(1):38-42. [DOI:10.1016/j.autrev.2010.08.015] [PMID]

[8] Sabry A, Elbasyouni SR, Sheashaa HA, Alhusseini AA, Mahmoud K, George SK, et al. Correlation between levels of TNF-alpha and IL-6 and hematological involvement in SLE Egyptian patients with lupus nephritis International Urology and Nephrology. 2006; 38(3-4):731-7. [DOI:10.1007/s11255006-0047-9] [PMID]

[9] Su DL, Lu ZM, Shen MN, Li X, Sun LY. Roles of pro-and anti-inflammatory cytokines in the pathogenesis of SLE. BioMed Research International. 2012; 2012(347141):1-15. [DOI:10.1155/2012/347141] [PMID] [PMCID]

[10] Wahren-Herlenius M, Dörner T. Immunopathogenic mechanisms of systemic autoimmune disease. The Lancet 2013; 382(9894):819-31. [DOI:10.1016/S0140-6736(13)60954-X]

[11] Lei HW, Wang JY, Dang QJ, Yang F, Liu X, Zhang JH, et al. Neuropsychiatric involvement in lupus is associated with the Nogo-a/NgR1 pathway. Journal of Neuroimmunology. 2017; 311:22-8. 
[12] Bertsias GK, Ioannidis JP, Aringer M, Bollen E, Bombardieri $\mathrm{S}$, Bruce IN, et al. EULAR recommendations for the management of systemic lupus erythematosus with neuropsychiatric manifestations: report of a task force of the EULAR standing committee for clinical affairs. Annals of the Rheumatic Diseases. 2010; 69(12):2074-82. [DOI:10.1136/ard.2010.130476] [PMID]

[13] Govoni M, Bortoluzzi A, Padovan M, Silvagni E, Borrelli $\mathrm{M}$, Donelli F, et al. The diagnosis and clinical management of the neuropsychiatric manifestations of lupus. Journal of $\mathrm{Au}-$ toimmunity. 2016; 74:41-72. [DOI:10.1016/j.jaut.2016.06.013] [PMID]

[14] Ahearn JM, Liu CC, Kao AH, Manzi S. Biomarkers for systemic lupus erythematosus. Translational Research. 2012; 159(4):326-42. [DOI:10.1016/j.trsl.2012.01.021] [PMID]

[15] Abda EA, Selim ZI, Radwan ME, Mahmoud NM, Herdan OM, Mohamad KA, et al. Markers of acute neuropsychiatric systemic lupus erythematosus: A multidisciplinary evaluation. Rheumatology International. 2013; 33(5):1243-53. [DOI:10.1007/s00296-012-2531-0] [PMID]

[16] Stanojcic M, Loheswaran G, Xu L, Hoffman SA, Sakic B. Intrathecal antibodies and brain damage in autoimmune MRL mice. Brain, Behavior, and Immunity. 2010; 24(2):289 97. [DOI:10.1016/j.bbi.2009.10.009] [PMID]

[17] Sato T, Fujii T, Yokoyama T, Fujita Y, Imura Y, Yukawa $\mathrm{N}$, et al. Anti-U1 RNP antibodies in cerebrospinal fluid are associated with central neuropsychiatric manifestations in systemic lupus erythematosus and mixed connective tissue disease. Arthritis \& Rheumatism. 2010; 62(12):3730-40. [DOI:10.1002/art.27700] [PMID]

[18] Plantinga L, Tift BD, Dunlop-Thomas C, Sam Lim S, Barrett Bowling C, Drenkard C. Geriatric assessment of physical and cognitive functioning in a diverse cohort of systemic lupus erythematosus patients: A pilot study. Arthrithis Care and Research. 2018; 70(10):1469-77. [DOI:10.1002/acr.23507] [PMID]

[19] Begenisic T, Spolidoro M, Braschi C, Baroncelli L, Milanese M, Pietra G, et al. Environmental enrichment decreases GABAergic inhibition and improves cognitive abilities, synaptic plasticity, and visual functions in a mouse model of Down syndrome. Frontiers in Cellular Neuroscience. 2011; 5:29. [DOI:10.3389/fncel.2011.00029] [PMID] [PMCID]

[20] Liu-Ambrose T, Nagamatsu LS, Graf P, Beattie BL, Ashe MC, Handy TC. Resistance training and executive functions: A 12-month randomized controlled trial. Archives of Internal Medicine. 2010; 170(2):170-8. [DOI:10.1001/archinternmed.2009.494] [PMID] [PMCID]

[21] Ercan E, Magro-Checa C, Valabregue R, Branzoli F, Wood ET, Steup-Beekman GM, et al. Glial and axonal changes in systemic lupus erythematosus measured with diffusion of intracellular metabolites. Brain. 2016; 139(5):1447-57. [DOI:10.1093/brain/aww031] [PMID] [PMCID]

[22] Best JR. Effects of physical activity on children's executive function: Contributions of experimental research on aerobic exercise. Developmental Review. 2010; 30(4):331-51. [DOI:10.1016/j.dr.2010.08.001] [PMID]

[23] Reis-Neto ET, Silva AE, Monteiro CM, Camargo LM, Sato EI. Supervised physical exercise improves endothelial function in patients with systemic lupus erythematosus. Rheuma- tology. 2013; 52(12):2187-95. [DOI:10.1093/rheumatology/ ket283] [PMID]

[24] Prado DM, Benatti FB, de Sá-Pinto AL, Hayashi AP, Gualano B, Pereira RM, et al. Exercise training in childhoodonset systemic lupus erythematosus: A controlled randomized trial. Arthritis Research \& Therapy. 2013; 15:R46. [DOI:10.1186/ar4205] [PMID] [PMCID]

[25] Lauenroth A, Ioannidis AE, Teichmann B. Influence of combined physical and cognitive training on cognition: A systematic review. BMC Geriatrics. 2016; 16:141. [DOI:10.1186/ s12877-016-0315-1] [PMID] [PMCID]

[26] Perandini LA, Sales-de-Oliveira D, Mello SB, Camara $\mathrm{NO}$, Benatti FB, et al. Exercise training can attenuate the inflammatory milieu in women with systemic lupus erythematosus. Journal of Applied Physiology. 2014; 117(6):639-47. [DOI:10.1152/japplphysiol.00486.2014] [PMID]

[27] Gleeson M, Bishop NC, Stensel DJ, Lindley MR, Mastana SS, Nimmo MA. The anti-inflammatory effects of exercise: Mechanisms and implications for the prevention and treatment of disease. Nature Reviews Immunology. 2011; 11(9):607-15. [DOI:10.1038/nri3041] [PMID]

[28] Golubović Š, Maksimović J, Golubović B, Glumbić N. Effects of exercise on physical fitness in children with intellectual disability. Research in Developmental Disabilities. 2012; 33(2):608-14. [DOI:10.1016/j.ridd.2011.11.003] [PMID]

[29] Baker LD, Frank LL, Foster-Schubert K, Green PS, Wilkinson CW, McTiernan A, et al. Effects of aerobic exercise on mild cognitive impairment: A controlled trial. Archives of Neurology. 2010; 67(1):71-9. [DOI:10.1001/archneurol.2009.307] [PMID] [PMCID]

[30] Abbott NJ, Patabendige AA, Dolman DE, Yusof SR, Begley DJ. Structure and function of the blood-brain barrier. Neurobiology of Disease. 2010; 37(1):13-25. [DOI:10.1016/j. nbd.2009.07.030] [PMID]

[31] Najarzadegan M, Nejati V, Amiri NA, Sharifian MA. [Effect of cognitive rehabilitation on executive function (working memory and attention) in children with attention deficit hyperactivity disorder (Persian)]. Journal of Rehabilitation Medicine. 2015; 4(2):97-108.

[32] Middleton LE, Ventura MI, Santos-Modesitt W, Poelke G, Yaffe K, Barnes DE. The Mental Activity and eXercise (MAX) trial: Effects on physical function and quality of life among older adults with cognitive complaints. Contemporary Clinical Trials. 2018; 64:161-6. [DOI:10.1016/j.cct.2017.10.009] [PMID] [PMCID]

[33] Lai L, Bruce H, Bherer L, Lussier M, Li KZ. Comparing the transfer effects of simultaneously and sequentially combined aerobic exercise and cognitive training in older adults. Journal of Cognitive Enhancement. 2017; 1(4):478-90. [DOI:10.1007/ s41465-017-0052-1] 